\title{
Influence of initial molecular substance on the diffusion flux across cell membranes
}

\author{
Bum Joon Jung ${ }^{1,2}$, Dae-Han $\mathrm{Ki}^{2}$ \\ ${ }^{1}$ Department of Biomedical Engineering, Rensselaer Polytechnic Institute, New York, USA \\ ${ }^{2}$ Proton Therapy Center, National Cancer Center, Goyang, South Korea \\ Email: jungb3@rpi.edu
}

Received 11 December 2013; revised 11 January 2014; accepted 22 January 2014

Copyright (C) 2014 Bum Joon Jung, Dae-Han Ki. This is an open access article distributed under the Creative Commons Attribution License, which permits unrestricted use, distribution, and reproduction in any medium, provided the original work is properly cited. In accordance of the Creative Commons Attribution License all Copyrights (C) 2014 are reserved for SCIRP and the owner of the intellectual property Bum Joon Jung, Dae-Han Ki. All Copyright (C) 2014 are guarded by law and by SCIRP as a guardian.

\section{ABSTRACT}

The influence of initial placement of molecular or ion substance is investigated on the diffusion fluxes across the cell membrane. The diffusion fluxes and recovery curves are obtained by considering both the singlespot and double-spot concentrations inside the cell membrane. The results show that the additional concentration inside the membrane reduces the net fluxes at the cell interior as well as the exterior. In addition, it is found that the change in diffusion flux at the two outer walls of the membrane by the two-spot concentrations in the cell membrane is weaker than that of the single-spot concentration at the center. The variation of the influence of initial locations of the molecular concentrations inside the cell membrane on the diffusion fluxes is also discussed. This result can be applied to the diffusion process in avascular collagenous tissues.

\section{KEYWORDS}

\section{Diffusion Flux; Cell Membrane; Recovery Curves}

\section{INTRODUCTION}

A diffusion process has been one of the most interesting processes in physical sciences since the diffusion provides physical insights into the flux variation in various scientific areas, such as biology, chemistry, mathematics, and physics. In biological fields, the diffusion process is used in animal dispersal, bacteria motion, cell movement, chemical diffusion such as drug release in membrane and tissues, and plant electrophysiology [1-8]. Especially, the theory of transport into or out of a cell by the intra-cellular diffusion has received considerable attention since this process has wide applications in biological sciences.
It has been shown that the diffusion in the human body is noteworthy over small distance scales on the order of $1-100 \mu \mathrm{m}$ and the diffusion plays an important role in the metabolic activity [9]. It has been also shown that the diffusion phenomena in cells would be strongly biased by the influence of environments. Hence, it is expected that the diffusion in a cell membrane involving double lipid layers would be significantly influenced by the location of the initial location of a molecular solute. Thus, in this paper we investigate the influence of initial placement of molecular substance on the diffusion fluxes at outer walls of the cell membrane by using the theoretical analysis with the boundary conditions since the diffusion processes are significant for the characterization of cellular and physiological processes and for the operation of biomedical machines [10], such as the heart-lung bypass device, kidney dialysis device, and membrane oxygenator. The diffusion fluxes and recovery curves in the cell membrane are obtained by considering the single-spot and double-spot concentrations, respectively, inside the cell membrane. The influence of initial location of the concentration inside the cell membrane on the net diffusion fluxes at the outer walls of the cell membrane is also discussed.

This paper is composed as follows. In Section 2, the diffusion equation in the cell membrane with the detailed initial and boundary conditions and the Danckwert's method for the diffusion-reaction equation are discussed. In Section 3, we obtain the solutions of the diffusion equation for the single-spot and double-spot concentrations inside the cell membrane and the recovery curves for both single-spot and double-spot concentrations. In Section 4 , we obtain the net flues at the wall boundary for the single-spot and double-spot concentrations inside the cell membrane. In Section 5, the influence of initial placement of molecular substance on the diffusion fluxes 
at outer walls of the cell membrane is discussed. In addition, we discuss the variations of the concentrations and recovery curves inside the cell membrane. Finally, the conclusions are given in Section 6.

\section{DIFFUSION IN THE CELL MEMBRANE}

From Fick's first and second equations for the flux $J(x, t)$, the diffusion equation [5] for the concentration $C(x, t)$ in the cell membrane is represented by

$$
\frac{\partial}{\partial t} C(x, t)=D \frac{\partial^{2}}{\partial x^{2}} C(x, t)
$$

where $x$ is the position in one-dimension, $t$ is the time, and $D$ is the diffusion coefficient. It is known that a typical value of the diffusion coefficient for ions such as $\mathrm{K}^{+}, \mathrm{Cl}^{-}$, and $\mathrm{Na}^{+}$is about $2.5 \times 10^{-6} \mathrm{~cm}^{2} / \mathrm{s}$ [11]. For the sake of the simplicity, we consider the one-dimensional diffusion problem for the investigation of the diffusion fluxes across cell membranes. In Equation (1), we retain only the diffusive flux term, but neglect the electrical drift effect since the influence of initial placement of molecular or ion substance on the diffusion fluxes at outer walls of the cell membrane is the main purpose of this work. Hence, we assume that the cell membrane has been localized in the domain of $0<x<L$ and the molecular concentrations at the domain boundaries as the boundary conditions are $C(0, t)=C_{0}$ and $C(L, t)=C_{L}$. Two cases for the initial intracellular concentration as the initial conditions shall be considered: $C(x, 0)=\left(C_{a} L\right) \delta(x-a)$ for the single-spot concentration case and $C(x, 0)=\left(C_{a} L\right) \delta(x-a)+\left(C_{b} L\right) \delta(x-b)$ for the double-spot concentration case, where $\delta(x)$ is the Dirac delta function and $a$ and $b$ are initial locations of the molecular concentrations. If the reaction phenomenon is involved in the diffusion process, the righthand side of Equation (1) would have an extra term proportional to the concentration: $-k C(x, t)$, where $k$ is a constant. In this case, the diffusion-reaction equation in the cell membrane is given by

$$
\frac{\partial}{\partial t} C(x, t)=D \frac{\partial^{2}}{\partial x^{2}} C(x, t)-k C(x, t)
$$

where $k C(x, t)$ represents the rate of removal of diffusing substance. It has been also shown that the diffusion-reaction equation is useful for the mathematical modeling of glioma growth and also for the investigation on the spreading of brain tumors [12] and $k C(x, t)$ term corresponds to the death of cells in the tumor growth model. It has been well known that the general solution of Equation (2) can also be obtained the following transformation based on Danckwert's method [13, 14]:

$$
C(x, t)=\mathrm{e}^{-k t} C_{1}(x, t)+k \int_{0}^{t} \mathrm{~d} t^{\prime} \mathrm{e}^{-k t^{\prime}} C_{1}\left(x, t^{\prime}\right),
$$

where $C_{1}(x, t)$ is the solution of Equation (1) with the same boundaries. As a result, the standard diffusion equation, Equation (1), is used to examine the change in diffusion fluxes at outer walls of the cell membrane depended the initial molecular substance. In the following Section III, the solutions, $C(x, t)$ for the single-spot and for the double-spot concentrations inside the cell membrane are discussed.

\section{SINGLE-AND DOUBLE-SPOT CONCENTRATIONS}

Using the separation of variables method, the solution of the diffusion equation for the domain $0<x<L$ would be represented by

$$
\begin{aligned}
C(x, t)= & C_{0}+\left(C_{L}-C_{0}\right) \frac{x}{L} \\
& +\sum_{n=1}^{\infty} A_{n} \sin \left(\lambda_{n} x\right) \exp \left(-D \lambda_{n}^{2} t\right),
\end{aligned}
$$

where $\lambda_{n}(=n \pi / L)$ is the separation constant determined by the boundary condition at $x=L, A_{n}$ are coefficients to be determined by the initial condition at $t=0$, and the first two terms $C_{0}+\left(C_{L}-C_{0}\right)(x / L)$ represents the solution when the separation constant is zero. Since we are interested in the effect of the initial molecular concentration $C(x, 0)$ inside the cell membrane, the coefficients $A_{n}$ are determined by

$$
A_{n}=\frac{2}{L} \int_{0}^{L} \mathrm{~d} x\left[C(x, 0)-C_{0}-\left(C_{L}-C_{0}\right) \frac{x}{L}\right] \times \sin \left(\frac{n \pi}{L} x\right),
$$

where $C(x, 0)$ would be, respectively, $\left(C_{a} L\right) \delta(x-a)$ for the single-spot concentration and

$\left(C_{a} L\right) \delta(x-a)+\left(C_{b} L\right) \delta(x-b)$ for the double-spot concentrations with $0<a<L$ and $0<b<L$. The consideration of the initial concentration inside the cell membrane would be also quite useful to understand the behavior of the tumor growth since it has been known that the spatial arrangement effect is crucial for the spatial spread of the cancerous cells and also for the appearance of the tumor in the human body [12]. Hence, the general solutions for the diffusion equations with the single- and double-spot concentrations are, respectively, found to be the following forms:

$$
\begin{aligned}
C_{S}(x, t)= & C_{0}+\left(C_{L}-C_{0}\right) \frac{x}{L} \\
& +2 \sum_{n=1}^{\infty}\left[C_{a} \sin \left(\frac{n \pi a}{L}\right)+\frac{1}{\pi} \frac{C_{L} \cos (n \pi)-C_{0}}{n}\right](6) \\
& \times \sin \left(\frac{n \pi x}{L}\right) \exp \left(-\frac{n^{2} \pi^{2}}{L^{2}} D t\right),
\end{aligned}
$$




$$
\begin{aligned}
C_{D}(x, t)= & C_{0}+\left(C_{L}-C_{0}\right) \frac{x}{L}+2 \sum_{n=1}^{\infty}\left[C_{a} \sin \left(\frac{n \pi a}{L}\right)\right. \\
& \left.+C_{b} \sin \left(\frac{n \pi b}{L}\right)+\frac{1}{\pi} \frac{C_{L} \cos (n \pi)-C_{0}}{n}\right] \\
& \times \sin \left(\frac{n \pi x}{L}\right) \exp \left(-\frac{n^{2} \pi^{2}}{L^{2}} D t\right) .
\end{aligned}
$$

Once the concentration function $C(x, 0)$ is obtained, the measurement of the concentration fluctuations would be determined by the signal correlation function $S(0) S(t)$ between the time at $t^{\prime}=0$ and $t^{\prime}=t$, where $S(t)=A \int \mathrm{d} x I(x) C(x, t), A$ is a factor related to the experimental detection, and $I(x)$ is the intensity of the laser beam [15]. A detailed investigation on the correlation spectroscopy for the influence of initial placement of molecular or ion substance on the concentration fluctuations will be treated elsewhere. The recovery curves for the single-spot $N_{S}(t)$ and two-spot $N_{D}(t)$ concentrations are then, respectively, obtained as follows:

$$
\begin{aligned}
& N_{S}(t)= \int_{0}^{L} \mathrm{~d} x C_{S}(x, t)=C_{0} L+\left(C_{L}-C_{0}\right) \frac{L}{2} \\
&-\frac{2 L}{\pi} \sum_{n=1}^{\infty}\left[C_{a} \sin \left(\frac{n \pi a}{L}\right)+\frac{1}{\pi} \frac{C_{L} \cos (n \pi)-C_{0}}{n}\right](8) \\
& \times\left(\frac{\cos (n \pi)-1}{n}\right) \exp \left(-\frac{n^{2} \pi^{2}}{L^{2}} D t\right) . \\
& N_{D}(t)=\int_{0}^{L} \mathrm{~d} x C_{D}(x, t) \\
&=C_{0} L+\left(C_{L}-C_{0}\right) \frac{L}{2}-\frac{2 L}{\pi} \sum_{n=1}^{\infty}\left[C_{a} \sin \left(\frac{n \pi a}{L}\right)\right. \\
&+\left.C_{b} \sin \left(\frac{n \pi b}{L}\right)+\frac{1}{\pi} \frac{C_{L} \cos (n \pi)-C_{0}}{n}\right] \\
& \times\left(\frac{\cos (n \pi)-1}{n}\right) \exp \left(-\frac{n^{2} \pi^{2}}{L^{2}} D t\right) .
\end{aligned}
$$

\section{NET DIFFUSION FLUX IN THE CELL MEMBRANE}

The diffusion fluxes at the wall boundaries $x=0$, i.e., $J_{0}$, and $x=L$, i.e., $J_{L}$, for the case of the single-spot concentration are, respectively, given by

$$
\begin{aligned}
J_{0}= & -\left.D \frac{\partial C_{S}}{\partial x}\right|_{x=0} \\
= & -\left(C_{L}-C_{0}\right) \frac{D}{L}-2 D \sum_{n=1}^{\infty}\left[C_{a} \sin \left(\frac{n \pi a}{L}\right)\right. \\
& \left.+\frac{1}{\pi} \frac{C_{L} \cos (n \pi)-C_{0}}{n}\right] \times\left(\frac{n \pi}{L}\right) \exp \left(-\frac{n^{2} \pi^{2}}{L^{2}} D t\right),
\end{aligned}
$$

$$
\begin{aligned}
J_{L}= & -\left.D \frac{\partial C_{S}}{\partial x}\right|_{x=L}=-\left(C_{L}-C_{0}\right) \frac{D}{L} \\
& -2 D \sum_{n=1}^{\infty}\left[C_{a} \sin \left(\frac{n \pi a}{L}\right)+\frac{1}{\pi} \frac{C_{L} \cos (n \pi)-C_{0}}{n}\right] \\
& \times\left(\frac{n \pi}{L}\right) \cos (n \pi) \exp \left(-\frac{n^{2} \pi^{2}}{L^{2}} D t\right)
\end{aligned}
$$

If we retain on the first term in the series, the net flux $\left|\Delta J_{S}\right|\left(\equiv\left|J_{L}-J_{0}\right|\right)$ for the single-spot concentration is then found to be

$$
\begin{aligned}
\left|\Delta J_{S}(a, t)\right|= & \left|\frac{4 D}{L}\left[\left(C_{L}-C_{0}\right)-C_{a} \pi \sin \left(\frac{\pi a}{L}\right)\right]\right| \\
& \times \exp \left(-\frac{\pi^{2}}{L^{2}} D t\right) .
\end{aligned}
$$

As it is seen in Equation (12), the net flux in the cell membrane is completely determined by the location $\sin (\pi a / L)$ and amount $C_{a}$ of the initial molecular concentration inside the cell membrane. Likewise, the diffusion fluxes at the wall boundaries $x=0$ and $x=L$ for the case of the double-spot concentrations are, respectively, obtained by

$$
\begin{aligned}
J_{0}= & -\left.D \frac{\partial C_{D}}{\partial x}\right|_{x=0}=-\left(C_{L}-C_{0}\right) \frac{D}{L} \\
& -2 D \sum_{n=1}^{\infty}\left[C_{a} \sin \left(\frac{n \pi a}{L}\right)+C_{b} \sin \left(\frac{n \pi b}{L}\right)\right. \\
& \left.+\frac{1}{\pi} \frac{C_{L} \cos (n \pi)-C_{0}}{n}\right] \times\left(\frac{n \pi}{L}\right) \exp \left(-\frac{n^{2} \pi^{2}}{L^{2}} D t\right), \\
J_{L}= & -\left.D \frac{\partial C_{D}}{\partial x}\right|_{x=L}=-\left(C_{L}-C_{0}\right) \frac{D}{L} \\
& -2 D \sum_{n=1}^{\infty}\left[C_{a} \sin \left(\frac{n \pi a}{L}\right)+C_{b} \sin \left(\frac{n \pi b}{L}\right)\right. \\
& \left.+\frac{1}{\pi} \frac{C_{L} \cos (n \pi)-C_{0}}{n}\right] \\
& \times\left(\frac{n \pi}{L}\right) \cos (n \pi) \exp \left(-\frac{n^{2} \pi^{2}}{L^{2}} D t\right)
\end{aligned}
$$

If we also retain on the first term as we did in Equation (12), the net flux $\left|\Delta J_{D}\right|\left(\equiv\left|J_{L}-J_{0}\right|\right)$ for the twospot concentrations is then obtained as

$$
\begin{aligned}
& \left|\Delta J_{D}(a, b, t)\right| \\
= & \mid \frac{4 D}{L}\left[\left(C_{L}-C_{0}\right)-C_{a} \pi \sin \left(\frac{\pi a}{L}\right)\right. \\
& \left.-C_{b} \pi \sin \left(\frac{\pi b}{L}\right)\right] \mid \exp \left(-\frac{\pi^{2}}{L^{2}} D t\right) .
\end{aligned}
$$


As shown in Equation (15), the net flux in the cell membrane is also completely controlled by the locations $\sin (\pi a / L)$ and $\sin (\pi b / L)$ and amounts $C_{a}$ and $C_{b}$ of the initial molecular concentrations inside the cell membrane.

Since the ion diffusion in biological membranes is one of the main transport processes [16], the results of $\left|\Delta J_{S}\right|$ and $\left|\Delta J_{D}\right|$, Equations (12) and (15), in this model analysis would provide useful information on the electric current and the membrane potential. Recently, the diffusional anisotropy has been investigated in avascular collagenous tissues since the diffusion in articular cartilage is the main molecular transport process [17]. Hence, the effects of the initial placement of molecular substance on the two-dimensional anisotropic diffusion process will be treated elsewhere.

\section{INFLUENCE OF INITIAL ION PLACEMENT}

In this section, the graph plotting for the 2D and 3D plots are obtained by the technical computing software $M a$ thematica. Figure 1 represents the net flux $\left|\Delta J_{S}\right|$ for the initial single-spot concentration case as a function of the scaled position $\bar{a}(\equiv a / L)$ for various values of the diffusion time $t$ when $C_{a}=\max \left\{C_{0}, C_{L}\right\}$. As it is seen, it is found that two non-flux positions for $\left|\Delta J_{S}\right|$ can be existed when the initial molecular concentration inside the cell membrane is equal to the larger one between two molecular concentrations at the domain boundaries. We have also found that that two non-flux positions are placed near the wall boundaries. In addition, it is found that the net flux $\left|\Delta J_{S}\right|$ decreases with an increase of the diffusion time $t$.

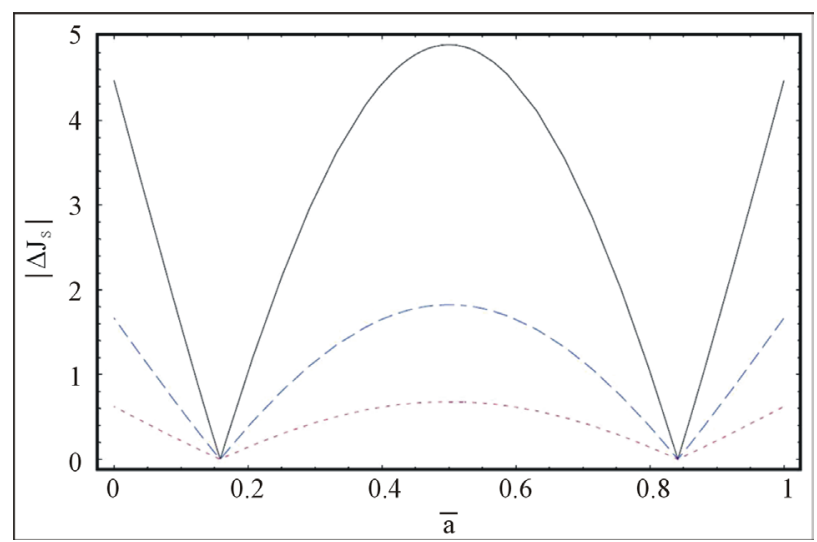

Figure 1. The net flux $\left|\Delta J_{S}\right|$ for the initial single-spot concentration case as a function of the scaled position $\bar{a}(\equiv a / L)$ when $C_{0}=2, C_{L}=1$, and $C_{a}=2$, i.e., $C_{a}=\max \left\{C_{0}, C_{L}\right\}$. The solid line represents the case of the scaled diffusion time $\bar{t}\left(\equiv t D / L^{2}\right)=0.1$. The dashed line represents the case of $\bar{t}=0.2$. The dotted line represents the case of $\bar{t}=0.3$.
Figure 2 shows the net flux $\left|\Delta J_{S}\right|$ for the initial single-spot concentration case as a function of the scaled position $\bar{a}$ for various values of the diffusion time $t$ when $C_{a}$ is smaller than $C_{0}$ and $C_{L}$. As shown in this figure, it is interesting to note that the non-flux positions for $\left|\Delta J_{S}\right|$ are disappeared inside the cell membrane when the initial molecular concentration inside the cell membrane is smaller than two molecular concentrations at the wall boundaries. In addition, the net flux $\left|\Delta J_{S}\right|$ is found to be quite small at the center of the cell membrane.

Figure 3 represents the scaled recovery curve $\bar{N}_{S}\left(\equiv N_{S} / L\right)$ for the initial single-spot concentration

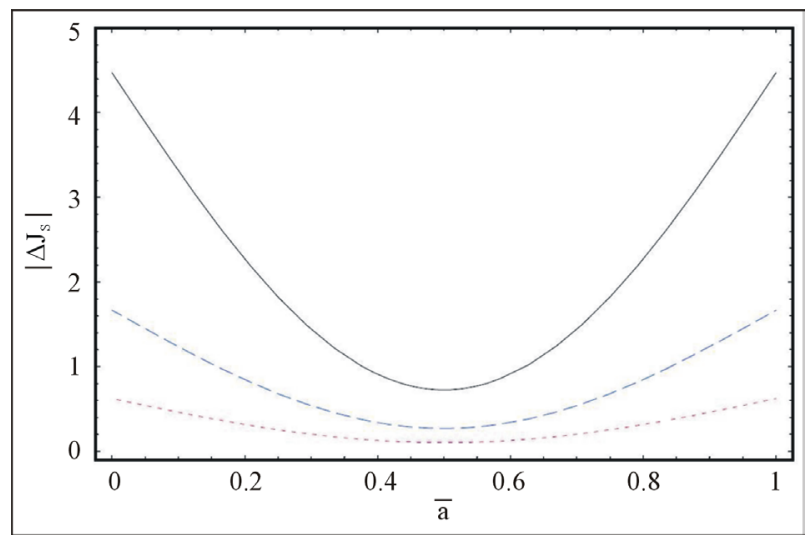

Figure 2. The net flux $\left|\Delta J_{s}\right|$ for the initial single-spot concentration case as a function of the scaled position $\bar{a}$ when $C_{0}=2, C_{L}=1$, and $C_{a}=0.8$, i.e., $C_{a}<\min \left\{C_{0}, C_{L}\right\}$. The solid line represents the case of the scaled diffusion time $\bar{t}=0.1$. The dashed line represents the case of $\bar{t}=0.2$. The dotted line represents the case of $\bar{t}=0.3$.

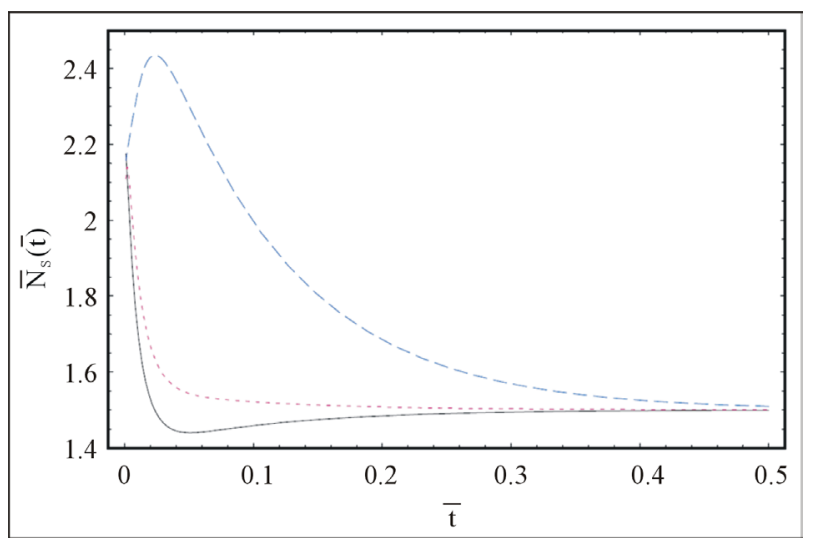

Figure 3. The scaled recovery curve $\bar{N}_{S}\left(\equiv N_{S} / L\right)$ for the initial single-spot concentration case as a function of the scaled diffusion time $\bar{t}$ when $C_{0}=2, C_{L}=1$, and $C_{a}=2$, i.e., $C_{a}=\max \left\{C_{0}, C_{L}\right\}$. The solid line represents the case of $\bar{a}=1 / 7$. The dashed line represents the case of $\bar{a}=1 / 2$. The dotted line represents the case of $\bar{a}=5 / 6$. 
case as a function of the scaled diffusion time $\bar{t}\left(\equiv t D / L^{2}\right)$ for various values of the initial location $\bar{a}$ when $C_{a}=\max \left\{C_{0}, C_{L}\right\}$. From this figure, it is found that the recovery curve $\bar{N}_{S}$ would have the maximum value when the initial single-spot concentration is placed near the right wall boundary and decreases with an increase of the diffusion time $\bar{t}$. However, we have found that the recovery curve $\bar{N}_{S}$ would have the minimum value when the initial single-spot concentration is placed near the left wall boundary and increases with an increase of the diffusion time $\bar{t}$. It is also found that that the recovery curve $\bar{N}_{S}$ decreases monotonically with an increase of the diffusion time when the initial single-spot concentration is placed at the center of the cell membrane.

Figure 4 shows the scaled recovery curve $\bar{N}_{S}$ for the initial single-spot concentration case as a function of the scaled diffusion time $\bar{t}$ for various values of the initial location $\bar{a}$ when $C_{a}=\min \left\{C_{0}, C_{L}\right\}$. As shown in this figure, it is found that the recovery curve $\bar{N}_{S}$ would have the minimum value when the initial single-spot concentration is placed near the left wall boundary at the center of the membrane and increases with an increase of the diffusion time $\bar{t}$. However, we have found that the recovery curve $\bar{N}_{S}$ has the maximum value when the initial single-spot concentration is placed near the right wall boundary and decreases with an increase of the diffusion time $\bar{t}$.

Figure 5 represents the surface plot of the net flux $\left|\Delta J_{D}\right|$ for the initial double-spot concentration case as a function of the scaled positions $\bar{a}(\equiv a / L)$ and $\bar{b}(\equiv b / L)$ when $C_{a}+C_{b}=\max \left\{C_{0}, C_{L}\right\}$. As we can see from this figure, it is found that two non-flux posi-

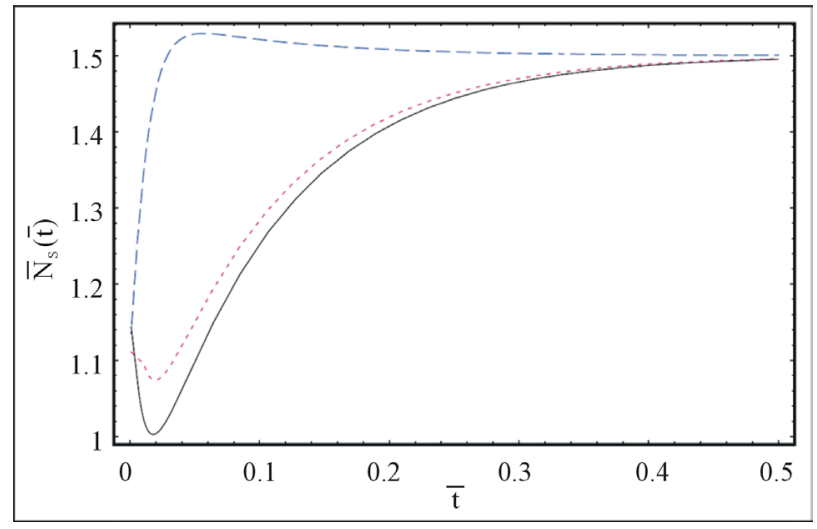

Figure 4. The scaled recovery curve $\bar{N}_{S}$ for the initial singlespot concentration case as a function of the scaled diffusion time $\bar{t}$ when $C_{0}=2, C_{L}=1$, and $C_{a}=1$, i.e., $C_{a}=\min \left\{C_{0}, C_{L}\right\}$. The solid line represents the case of $\bar{a}=1 / 7$. The dashed line represents the case of $\bar{a}=1 / 2$. The dotted line represents the case of $\bar{a}=5 / 6$. tions for $\left|\Delta J_{\underline{D}}\right|$ can be existed for the scaled position $\bar{a}$ for a given $\bar{b}$ and also for the scaled position $\bar{b}$ for a given $\bar{a}$. It is also found that the two non-flux positions are located near the wall boundaries. In addition, the net flux $\left|\Delta J_{D}\right|$ has the maximum value at the center of the cell membrane.

Figure 6 represents the surface plot of the net flux $\left|\Delta J_{D}\right|$ for the initial double-spot concentration case as a function of the scaled positions $\bar{a}$ and $\bar{b}$ when the summation of concentrations $C_{a}+C_{b}$ is smaller than $C_{0}$ and $C_{L}$. As it is seen in this figure, it is found that the non-flux positions for $\left|\Delta J_{D}\right|$ are disappeared inside the cell membrane when the initial total molecular con-

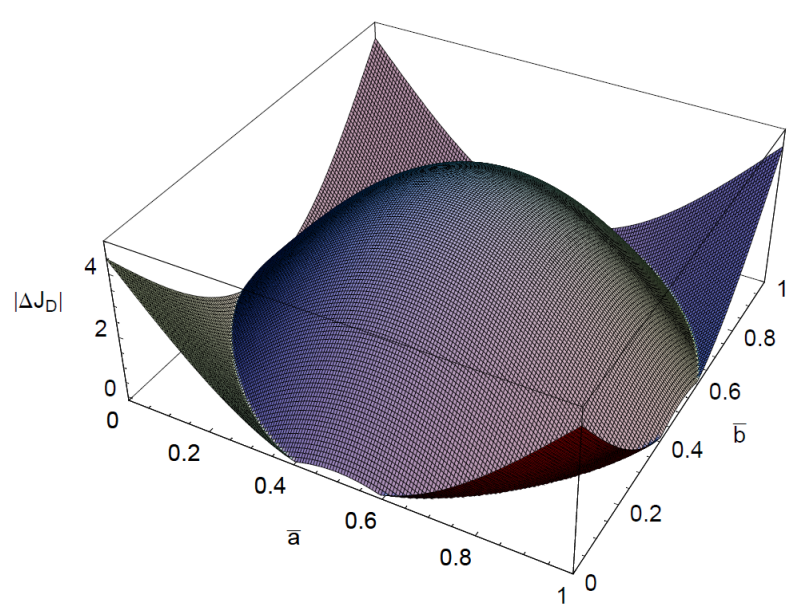

Figure 5. The surface plot of the net flux $\left|\Delta J_{D}\right|$ for the initial double-spot concentration case as a function of the scaled positions $\quad \bar{a}(\equiv a / L)$ and $\bar{b}(\equiv b / L)$ when $\bar{t}=0.1, \quad C_{0}=2$, $C_{L}=1, C_{a}=1$, and $C_{b}=1$, i.e., $C_{a}+C_{b}=\max \left\{C_{0}, C_{L}\right\}$.

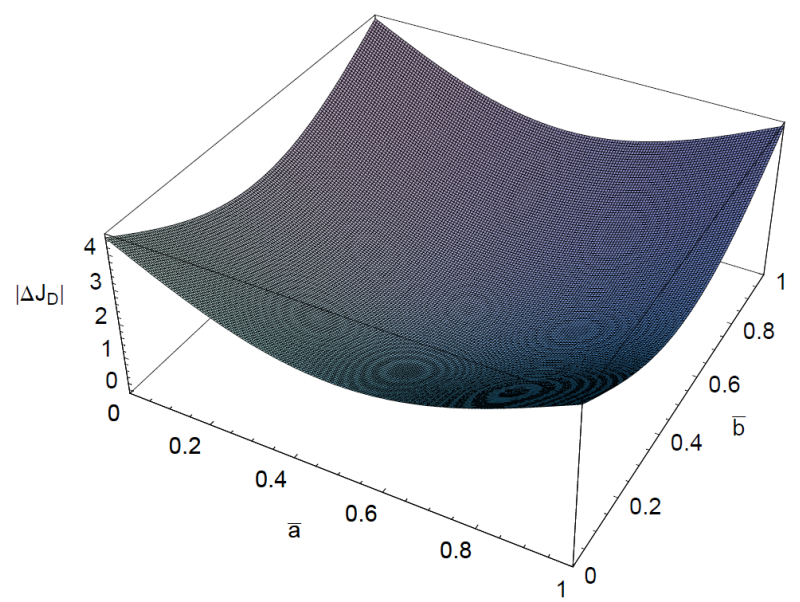

Figure 6. The surface plot of the net flux $\left|\Delta J_{D}\right|$ for the initial double-spot concentration case as a function of the scaled positions $\bar{a}$ and $\bar{b}$ when $\bar{t}=0.1, C_{0}=2, C_{L}=1, C_{a}=0.4$, and $C_{b}=0.4$, i.e., $C_{a}+C_{b}<\min \left\{C_{0}, C_{L}\right\}$. 
centration $C_{a}+C_{b}$ inside the cell membrane is smaller than two molecular concentrations $C_{0}$ and $C_{L}$ at the wall boundaries. The net flux $\left|\Delta J_{D}\right|$ for the initial double-spot concentration case is found to be also quite small at the center of the cell membrane.

Figure 7 shows the scaled recovery curve $\bar{N}_{D}$ for the initial double-spot concentration case as a function of the scaled diffusion time $\bar{t}$ for various values of the scaled positions $\bar{a}$ and $\bar{b}$ when $C_{a}+C_{b}=\max \left\{C_{0}, C_{L}\right\}$. As it is seen in this figure, the recovery curves $\bar{N}_{D}$ have maximum values at very short diffusion times $\bar{t}$. It is also found that the maximum time of the recovery curve $\bar{N}_{D}$ has been increased as the two positions are getting close to the center of the cell membrane.

Figure 8 represents the scaled recovery curve $\bar{N}_{D}$ for the initial double-spot concentration case as a function of the scaled diffusion time $\bar{t}$ for various values of the scaled positions $\bar{a}$ and $\bar{b}$ when

$C_{a}+C_{b}=\min \left\{C_{0}, C_{L}\right\}$. From this figure, we have found that the recovery curve $\bar{N}_{D}$ has no maximum and increases and finally saturates with increasing diffusion time $\bar{t}$ when the two locations $\bar{a}$ and $\bar{b}$ are away from the center of the membrane. However, it is found that the recovery curve $\bar{N}_{D}$ has the maximum value at the small diffusion time and saturates with an increase of the diffusion time $\bar{t}$.

\section{CONCLUSIONS}

In this work, we inspected the influence of initial placement of molecular or ion substance on the diffusion fluxes at outer walls of the cell membrane. The recovery curves in the cell membrane were obtained for the single-

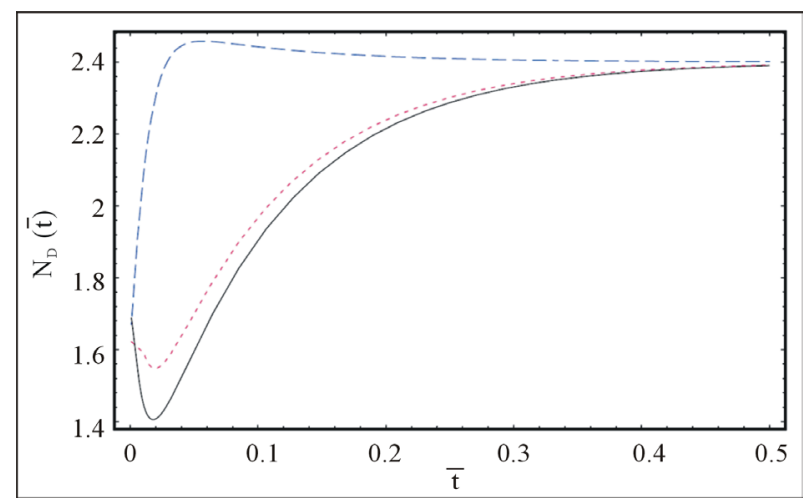

Figure 7. The scaled recovery curve $\bar{N}_{D}$ for the initial double-spot concentration case as a function of the scaled diffusion time $\bar{t}$ when $\bar{t}=0.1, C_{0}=2, C_{L}=1, C_{a}=1$, and $C_{b}=1$, i.e., $C_{a}+C_{b}<\max \left\{C_{0}, C_{L}\right\}$. The solid line represents the case of $\bar{a}=1 / 4$ and $\bar{b}=3 / 4$. The dashed line represents the case of $\bar{a}=4 / 9$ and $\bar{b}=5 / 9$. The dotted line represents the case of $\bar{a}=1 / 3$ and $\bar{b}=2 / 3$.

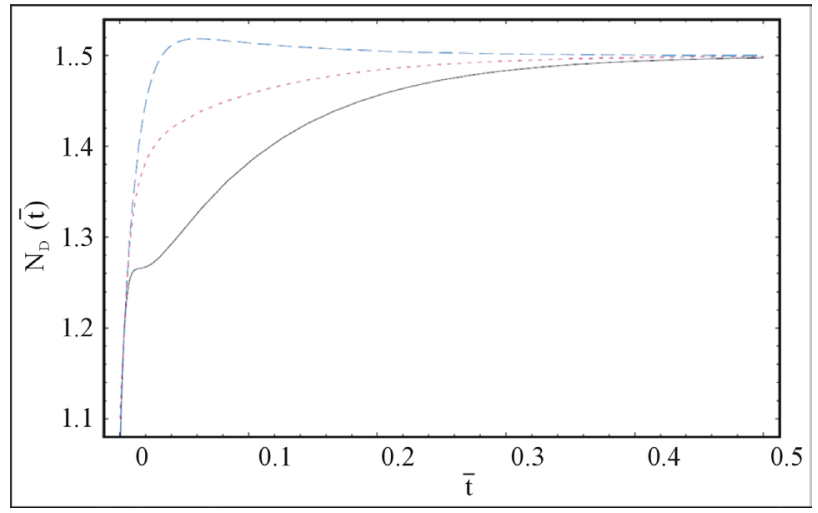

Figure 8. The scaled recovery curve $\bar{N}_{D}$ for the initial double-spot concentration case as a function of the scaled diffusion time $\bar{t}$ when $\bar{t}=0.1, C_{0}=2, C_{L}=1, C_{a}=0.5$, and $C_{b}=0.5$, i.e., $C_{a}+C_{b}<\min \left\{C_{0}, C_{L}\right\}$. The solid line represents the case of $\bar{a}=1 / 4$ and $\bar{b}=3 / 4$. The dashed line represents the case of $\bar{a}=4 / 9$ and $\bar{b}=5 / 9$. The dotted line represents the case of $\bar{a}=1 / 3$ and $\bar{b}=2 / 3$.

spot and double-spot concentrations, respectively. The net diffusion fluxes were obtained by considering both single-spot and double-spot concentrations across the cell membrane. For the initial single-spot concentration case, it was found that two non-flux positions would exist near the wall boundaries if the initial molecular concentration inside the cell membrane were equal to the larger one between the two molecular concentrations at the domain boundaries. It is also found that the two non-flux positions were placed near the center of the cell membrane when the initial molecular concentration inside the cell membrane was equal to the smaller one between the two molecular concentrations at the wall boundaries. In addition, we have found that the non-flux positions disappeared inside the cell membrane when the initial molecular concentration was smaller than two molecular concentrations at the wall boundaries. The molecular concentration $C_{S}$ could be increased more than $C_{0}$ when the initial single-spot concentration was placed near the left wall boundary and $C_{a}=\max \left\{C_{0}, C_{L}\right\}$. However, we have found that the molecular concentration monotonically decreases with an increase of the position when the initial single-spot concentration is placed at the center or near the right wall boundary. Moreover, we have found that the molecular concentration can be smaller than $C_{L}$ when the initial single-spot concentration was placed near the left wall boundary. Thus, the recovery curve would have the maximum vale when $C_{a}=\max \left\{C_{0}, C_{L}\right\}$ and the initial single-spot concentration was placed near the right wall boundary and the recovery curve decreases with an increase of the diffusion time. The minimum value would be when $C_{a}=\min \left\{C_{0}, C_{L}\right\}$ and the initial 
single-spot concentration was placed near the left wall boundary at the center of the membrane and increases with an increase of the diffusion time. For the initial double-spot concentration case, it was found that two non-flux positions were located near the wall boundaries when $C_{a}+C_{b}=\max \left\{C_{0}, C_{L}\right\}$. It is also found that the net flux had the maximum value at the center of the cell membrane. We found two non-flux positions could also be existed near the center of the cell membrane when $C_{a}+C_{b}=\min \left\{C_{0}, C_{L}\right\}$ and the net flux was still quite small at the center of the cell membrane. However, we have found that the non-flux positions were disappeared inside the cell membrane when $C_{a}+C_{b}<\min \left\{C_{0}, C_{L}\right\}$ and the net flux for the initial double-spot concentration case was found to be also quite small at the center of the cell membrane. We have found that the molecular concentration $C_{D}$ had the maximum value when two positions were near the center of the cell membrane when $C_{a}+C_{b}=\max \left\{C_{0}, C_{L}\right\}$. However, we have found that the molecular concentration decreased with an increase of the distance $\bar{x}$ regardless of the locations of the initial double-spot concentrations when

$C_{a}+C_{b}=\min \left\{C_{0}, C_{L}\right\}$. It is found that the recovery curves had maximum values at very short diffusion times and the recovery curve increased as the two positions were getting close to the center of the cell membrane when $C_{a}+C_{b}=\max \left\{C_{0}, C_{L}\right\}$. It is found that the recovery curve at first had no maximum, but increased and finally saturated with increasing diffusion time when the two initial locations were away from the center of the membrane. It had the maximum value at the small diffusion time and saturated with an increase of the diffusion time when $C_{a}+C_{b}=\min \left\{C_{0}, C_{L}\right\}$. From this work, we have found that the influence of initial locations and amounts of the molecular substance plays a significant role on the diffusion fluxes across the cell membrane. Since the results are obtained in the analytic expressions with the appropriate physical conditions, the equations in this work would be quite reliable for understanding the diffusion process in biological systems. Hence, the results of this work would be useful for understanding the characteristics and properties of the intracellular diffusion process as well as the spread of the cancerous cell and appearance of the tumor. The results in this work can also be applied to the enhancement of the diffusion process in articular cartilage. Moreover, these results would be applied to the biomedical machines related to the biological diffusion processes including the initial ion concentrations inside the wall boundaries.

\section{ACKNOWLEDGEMENTS}

The authors gratefully acknowledge Prof. Y.-K. Lim for useful discussions and encouragements while visiting the Proton Therapy Center at National Cancer Center of South Korea. This research was initiated while one of the authors (B. J. Jung) from Rensselaer Polytechnic Institute, USA was affiliated with the Proton Therapy Center at National Cancer Center as a Summer Intern Scholar.

\section{REFERENCES}

[1] Berg, H.C. and Purcell, E.M. (1967) A method for separating according to mass a mixture of macromolecules or small particles suspended in a fluid, I. Theory. Proceedings of the National Academy of Sciences USA, 58, 862869. http://dx.doi.org/10.1073/pnas.58.3.862

[2] Berg, H.C. and Purcell, E.M. (1977) Physics of chemoreception. Biophysical Journal, 20, 193-219. http://dx.doi.org/10.1016/S0006-3495(77)85544-6

[3] Iwasa, Y. and Teramoto, E. (1984) Branching-diffusion model of the formation of a population's distributional pattern. Journal of Mathematical Biology, 19, 109-124. http://dx.doi.org/10.1007/BF00275934

[4] Berg, H.C. (1993) Random walks in biology. Expanded Edition, Princeton University Press, Princeton.

[5] Jones, D.S. and Sleeman, B.D. (2000) Differential equations and mathematical biology. Chapman \& Hall, London.

[6] Murray, J.D. (2001) Mathematical biology, vol. I: An introduction. 3rd Edition, Springer, Berlin.

[7] Caputo, M. and Cametti, C. (2007) Diffusion with memory in two cases of biological interest. Journal of Theoretical Biology, 254, 697-703. http://dx.doi.org/10.1016/j.jtbi.2008.06.021

[8] Niklas, K.J. and Spatz, H.-C. (2012) Plant physics. The University of Chicago Press, Chicago. http://dx.doi.org/10.7208/chicago/9780226586342.001.00 $\underline{01}$

[9] Herman, I.P. (2008) Physics of human body. Springer, Berlin.

[10] Truskey, G.A., Yuan, F. and Katz, D.F. (2004) Transport phenomena in biological systems. Pearson Prentice Hall, Upper Saddle River.

[11] Ermentrout, G.B. and Terman, D.H. (2010) Mathematical foundations of neuroscience. Springer, Berlin. http://dx.doi.org/10.1007/978-0-387-87708-2

[12] Murray, J.D. (2003) Mathematical biology, vol. II: Spatial models and biomedical applications. 3rd Edition, Springer, Berlin.

[13] Danckwerts, P.V. (1951) Absorption by simultaneous diffusion and chemical reaction into particles of various shapes and into falling drops. Transactions of the faraday society, 47, 1014-1023. http://dx.doi.org/10.1039/tf9514701014

[14] Crank, J. (1975) The mathematics of diffusion. 2nd Edition. Oxford University Press, Oxford.

[15] Jackson, M.B. (2006) Molecular and cellular biophysics. Cambridge University Press, Cambridge. http://dx.doi.org/10.1017/CBO9780511754869

[16] Scherer, P.O.J. and Fischer, S.F. (2010) Theoretical molecular biophysics. Springer, Berlin. 
http://dx.doi.org/10.1007/978-3-540-85610-8

[17] Leddy, H.A., Haider, M.A. and Guilak, F. (2006) Diffusional anisotropy in collagenous tissues: Fluorescence imaging of continuous point photobleaching. Biophysical

Journal, 91, 311-316.

http://dx.doi.org/10.1529/biophysj.105.075283 